\title{
Fatores que influenciam no nível de qualidade de vida medida pela escala WHOQOL-BREF e tendência suicida em uma cadeia feminina do estado de São Paulo, Brasil
}

Factors that influence the level of quality of life measured

by the WHOQOL-BREF scale and suicide trend in a female chain in the state of São Paulo, Brazil

Factores que influyen en el nivel de calidad de vida medido por la escala WHOQOL-BREF y tendencia suicida en una cadena femenina en el estado de São Paulo, Brasil

Eduardo Henrique Teixeira https://orcid.org/0000-0003-1376-5080

Rogério Gomes da Silva Júnior - https://orcid.org/0000-0002-2082-0773

Giuliana Perrotte - https://orcid.org/0000-0003-3893-1743

Maria Lígia de Carvalho Solssia - https://or cid.org/0000-0001-6256-

$\underline{2330}$

\section{RESUMO:}

Introdução: A população de mulheres presas tem aumentado nas últimas décadas em todo o mundo, o Brasil ocupa a $4^{a}$ posição em número de mulheres presas e as taxas de depressão e risco de suicídio são elevadas. Objetivo: Avaliar o perfil sociodemográfico da população de uma prisão feminina, bem como fatores que influenciam o nível de qualidade de vida e aspectos da saúde mental. Método: Foram utilizados dois questionários. Um instrumento com questões demográficas e psicopatológicas gerais e a escala WHOQOL-BREF para avaliar o nível de qualidade de vida. Resultados: A amostra estudada foi de 214 presas. Na escala WHOQOLBREF, os escores de qualidade de vida foram baixos: "precisa melhorar" e "regular". Comportamento suicida, quando comparado a outros estudos, foi elevado nessa população e suporte psicológico ou psiquiátrico era raros. As visitas tiveram impacto no escore do domínio psicológico do WHOQOLBREF e promoveram um efeito protetor para comportamento suicida. Conclusão: É precário o suporte em saúde mental e o risco do isolamento 
compromete a saúde mental das presas. É fundamental o contato com o exterior, entretanto, ainda existem várias barreiras e limitações.

Palavras chave: Prisão feminina, presas, saúde mental, qualidade de vida, ideação suicida, tentativa de suicídio, visitas, WHOQOL-BREF

\section{ABSTRACT:}

Introduction: The population of women prisoners has increased in recent decades worldwide and Brazil occupies the 4th position in number of women prisoners and the rates of depression and risk of suicide are high. Objective: To evaluate the sociodemographic profile of the population of a female prison, as well as factors that influence the level of quality of life and aspects of mental health. Method: Two questionnaires were used. An instrument with general demographic and psychopathological issues and the WHOQOL-bref scale to assess the level of quality of life. Results: The sample studied was 214 women. On the WHOQOL-BREF scale, the quality of life score was low: "needs to improve" and "regular". Suicidal behavior, when compared to other studies, was high in this population and psychological or psychiatric support was rare. The visits had an impact on the WHOQOL-BREF psychological domain score and promoted a protective effect against suicidal behavior. Conclusion: Mental health support is precarious, and the risk of isolation compromises the mental health of prisoners. Contact with the outside is essential, however, there are still several barriers and limitations.

Keywords: Female prison, female prisoners, mental health, quality of life, suicidal ideation, suicide attempt, visits, WHOQOL-BREF

\section{RESUMEN:}

Introducción: La población de mujeres presas ha aumentado en las últimas décadas a nivel mundial y Brasil ocupa la $4^{a}$ posición en número de mujeres presas y las tasas de depresión y riesgo de suicidio son altas. Objetivo: Evaluar el perfil sociodemográfico de la población de un centro penitenciario femenino, así como los factores que influyen en el nivel de calidad de vida y aspectos de salud mental. Método: Se utilizaron dos cuestionarios. Un instrumento con cuestiones demográficas y psicopatológicas generales y la escala WHOQOL-bref para evaluar el nivel 
de calidad de vida. Resultados: La muestra estudiada fue de 214 mujeres. En la escala WHOQOL-BREF, el puntaje de calidad de vida fue bajo: "necesita mejorar" y "regular". La conducta suicida, en comparación con otros estudios, fue alta en esta población y el apoyo psicológico o psiquiátrico fue raro. Las visitas tuvieron un impacto en la puntuación del dominio psicológico WHOQOL-BREF y promovieron un efecto protector contra el comportamiento suicida. Conclusión: El apoyo a la salud mental es precario y el riesgo de aislamiento compromete la salud mental de los reclusos. El contacto con el exterior es fundamental, sin embargo, todavía existen varias barreras y limitaciones.

Palabras clave: prisión femenina, reclusas, salud mental, calidad de vida, ideación suicida, intento de suicidio, visitas, WHOQOL-BREF

Como citar: Teixeira EH, Silva Júnior RG, Perrotte G, Solssia MLC Fatores que influenciam no nível de qualidade de vida medida pela escala WHOQOL-BREF e tendência suicida em uma cadeia feminina do estado de São Paulo, Brasil. Debates em Psiquiatria, Rio de Janeiro, 2021; 11:1-23. https://doi.org/10.25118/2763-9037.2021.v11.191

Conflito de interesses: declaram não haver

Fonte de financiamento: declaram não haver

Parecer CEP: PUC-Campinas (Parecer: 2.209.813) e da Secretaria de Administração Penitenciária do estado de São Paulo (Parecer: 2.438.660)

Recebido em: 14/07/2021

Aprovado em: $15 / 12 / 2021$

Publicado em: 30/12/2021

\section{Introdução}

Segundo as diretrizes estabelecidas no Primeiro Congresso das Nações Unidas sobre a Prevenção do Crime e o Tratamento dos Delinquentes, realizado em Genebra em 1955, todo cidadão recluso no sistema penitenciário tem seus direitos definidos em relação ao local de reclusão, espaço físico, alimentação, vestimenta e atendimento à sua saúde [1] . Trata-se de uma população com características específicas, em uma 
situação de vulnerabilidade e risco, que deve receber abordagem ampla dentro dos princípios da confidencialidade, consentimento, honestidade e busca por objetividade e qualificações, segundo Diretrizes Éticas para a Prática da Psiquiatria Forense da Academia Americana de Psiquiatria [2] .

Estudo epidemiológico amplo realizado recentemente nos Estados Unidos para avaliar as condições de saúde física e mental entre encarcerados identificou índices significativamente maiores de pessoas doentes quando comparados à população geral. Este estudo recomendou investimentos em relação aos tratamentos na área de saúde, principalmente com doentes mentais e dependentes químicos, como parte fundamental do processo de reinserção do indivíduo à sociedade []ㅡ.

Especificamente em relação às mulheres presas, nas Regras das Nações Unidas constam orientações para o tratamento e medidas não privativas de liberdade para mulheres infratoras (Regras de Bangkok), as quais visam uma assistência digna e possibilidade de recuperação e inserção social plenas [4]. São estabelecidas estratégias específicas para o gênero, como atendimento ginecológico, tratamento de doenças sexualmente transmissíveis, gravidez, amamentação, entre outras.

As Regras de Bangkok norteiam como devem ser os serviços prisionais direcionados às características e demandas específicas das mulheres. Por exemplo, de acordo com a regra 4, sobre alocação, as mulheres devem permanecer preferencialmente em prisões próximas a seus locais de origem $[\underline{4}, \underline{5}]$.

Apesar da mudança recente na Lei de Execuções Penais no Brasil promovida pela Lei no $11.942 / 2009$ []], a qual aborda as condições mínimas de assistência às mães sobre regime de privação de liberdade, em nosso país, algumas das regras de Bangkok, na maioria dos presídios femininos brasileiros, não são possíveis de serem cumpridas. Grande parte desses presídios eram penitenciárias masculinas que foram "adaptadas" para receber mulheres infratoras.

Da mesma forma que acontece em outros países []], a disponibilidade de ferramentas e serviços direcionados para mulheres presas no Brasil é inconsistente e baseados nos modelos e normas aplicadas aos homens [ㅈ]. Tais desigualdades exigem uma revisão da lente tradicionalmente centrada no homem para a modernização e uma medida de reclusão digna [ㅁ] . 
A população carcerária de mulheres presas vem aumentando progressivamente nas últimas décadas ao redor do mundo. Foi fortemente relacionado ao combate ao tráfico de drogas, mas também decorrente de outras questões, pois atingiu mais mulheres de baixa renda e não brancas $[\underline{10}, \underline{11}]$.

O Brasil faz parte do grupo de doze países com maior população prisional feminina do mundo. Ocupa a $4^{a}$ posição em população absoluta, ficando atrás apenas dos Estados Unidos, da China e da Rússia. Quanto à taxa de aprisionamento, que representa o número de mulheres presas por 100 mil habitantes, o Brasil está em terceiro lugar, atrás apenas dos Estados Unidos e da Tailândia [12].

No Brasil, que atualmente tem a maior população carcerária da América do Sul, em 2016 tinha 726.712 presos, sendo 42.355 mulheres [10, 13]. O estado de São Paulo tem a maior população entre os estados brasileiros, não para de crescer e corresponde a $36 \%$ de todos as presas do país (15.104 presos), apesar de ficar apenas em $6^{\circ}$ lugar quando avaliada a taxa de aprisionamento por 100 mil habitantes, conforme dados do relatório divulgado pelo Ministério da Justiça referentes ao primeiro semestre de [12-14].

A taxa de aprisionamento de brasileiras apresentou um aumento incomparável com o grupo dos países com maior número de encarceradas. No período de 2000 e 2016, esta taxa aumentou em $455 \%$ no país [ 12 , 13]. Já a Rússia, no mesmo período, diminuiu em $2 \%$ o aprisionamento de tal população [9]].

Estudos em relação à população carcerária especificamente do sexo feminino são necessários e precisam ser aprofundados, considerando o aumento de prisões entre mulheres em todo mundo [15] , além da falta de recursos adequados e específicos ao gênero.

Revisão sistematizada sobre doença mental entre prisioneiros no mundo todo ( $n=33.588$ ) encontrou maior prevalência de transtorno mentais entre presas quando comparadas aos homens e uma tendência de aumento maior nas taxas de transtornos do tipo depressivo entre mulheres [16]. 0 mesmo padrão de resultado foi encontrado em um estudo com a população carcerária feminina do Rio de Janeiro, estado do Rio de Janeiro, Brasil, no qual foram avaliadas quarenta mulheres [17]. Estudo também usando 
metodologia semelhante com uma população carcerária feminina da cidade de Campinas, estado de São Paulo, Brasil, realizado entre 2012 e 2013, utilizando a escala SFR com $n=1.013$ mulheres presas, encontrou taxa de $66 \%$ de transtornos mentais [18].

As taxas de transtorno depressivo entre as presas são expressivamente maiores em relação à taxa de depressão na população geral [19]. Na Alemanha, estudo com presas encontrou uma taxa cinco vezes maior de suicídio comparado à população geral [20], apesar do suicídio ainda ser mais frequente entre homens presos [21].

Segundo levantamento do governo brasileiro, dentre uma população de presas $(n=27.029)$, foram registradas 48,2 mortes auto provocadas para cada 100 mil mulheres entre a população prisional, enquanto esse número para a população total foi de 2,3 suicídios registrados para cada grupo de $100 \mathrm{mil}$ mulheres [22] . Com esses valores estima-se que as chances de uma mulher se suicidar são até 20 vezes maiores entre a população prisional, quando comparada à população brasileira feminina total [23].

Segundo último relatório da OMS, a taxa de mortalidade por suicídio no mundo é de 10.6 e o Brasil ainda mantém tendência de aumento crescente nas taxas [24, 25] . O suicídio é um fenômeno complexo [26] e as pessoas em condições vulneráveis devem ser foco de ações preventivas dos serviços de saúde. Sabe-se também que o estresse crônico e o isolamento social, presentes no ambiente carcerário, estão entre os fatores de risco para o suicídio $[\underline{27}, \underline{28}]$.

Segundo estudo comparando prisioneiros homens com mulheres realizado na Inglaterra com $n=6453$, confirmou a importância dos relacionamentos afetivos na saúde mental das mulheres muito mais do que entre os homens [르]. A visita nos presídios é um fator importante para que as mulheres mantenham seus vínculos com familiares e amigos. Muitas vezes, é prejudicada por motivos como localização geográfica da prisão, questões

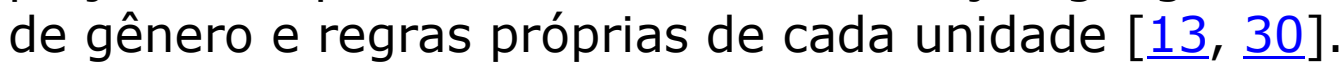

O nível de qualidade de vida de uma pessoa, segundo OMS, é definida como a percepção do indivíduo de sua posição na vida no contexto da cultura e sistema de valores nos quais ele vive e em relação aos seus objetivos, expectativas, padrões e preocupações. Alguns instrumentos foram desenvolvidos para avaliar este item e podem ajudar dando uma 
visão geral de uma população e comparar com outros grupos e ambientes [31].

Estudos com populações específicas, utilizando a escala para avaliação de qualidade de vida, WHOQOL-BREF, encontraram pontuação baixa em populações específicas, como em populações acometidas por doenças crônicas, como estudo realizado com pessoas contaminadas com HIV e estudo com pacientes em diálise devido a insuficiência renal $[\underline{32}, \underline{33}]$, além de refugiados [34] e idosos com limitações [35].

Considerando o aumento progressivo no número de presas e a grave situação do sistema prisional no Brasil, é fundamental uma avaliação cuidadosa desta população para que medidas possam ser definidas de forma mais efetivas. Neste sentido, no estudo abordaremos aspectos da saúde mental das presas, além de fatores relativos ao nível de qualidade de vida das mesmas.

\section{Método}

Trata-se de um estudo descritivo e transversal para avaliar fatores que influenciam a qualidade de vida de presas da Cadeia Feminina de Campinas, do estado de São Paulo, Brasil, além de avaliar aspectos da saúde mental, do suicídio e a relação com o padrão de visitas na prisão.

Foram utilizados dois questionários: um geral elaborado pela equipe de pesquisa e a escala autoaplicável WHOQOL-BREF, padronizada pela OMS (Organização Mundial da Saúde). Essa última é uma versão reduzida da escala WHOQOL-100 (Worth Health Organization Quality of Life), que surgiu pela necessidade de um instrumento curto que pudesse ser rapidamente preenchido e ao mesmo tempo representar características psicométricas satisfatórias [36]. Questionário com respostas incompletas maior que $20 \%$ foram desconsiderados.

Um questionário geral elaborado pela equipe consistia em 20 questões gerais diversas, que abordaram aspectos epidemiológico, como estado civil e escolaridade, tratamentos psicológico e psiquiátrico, ideação suicida e tentativa de suicídio, recebimento de visitas, uso de drogas, entre outros. Não foi usada escala padronizada para avaliação de padrão suicida porque não fazia parte do protocolo da pesquisa incialmente.

A escala WHOQOL-BREF é pautada no conceito de qualidade de vida da OMS (1995), que é "a percepção do indivíduo de sua inserção na vida no 
contexto da cultura e sistemas de valores nos quais ele vive e em relação aos seus objetivos, expectativas, padrões e preocupações". É composta por 26 questões, sendo duas sobre qualidade de vida de maneira geral e as demais 24 representando facetas que compõem de fato o instrumento de avaliação. As facetas compõem 4 domínios: os domínios físico, psicológico, relações sociais e meio ambiente. Foi validada no Brasil em 2000 [37].

A pesquisa foi realizada de 02/2018 a 08/2018, nas dependências da cadeia. Em decorrência da estrutura física e da dinâmica da própria instituição, não foi possível um encontro com todas as presas, apesar de todas serem convidadas. Sendo assim, inicialmente os pesquisadores reuniram-se com presas denominadas "multiplicadoras", as quais preencheram seus questionários e, após um treinamento, levaram as escalas para serem aplicadas nas demais presas.

As presas denominadas "multiplicadoras" eram reconhecidas como presas de melhor formação escolar e com mais habilidades no relacionamento com outras presas. Geralmente elas já eram responsáveis pelos seus respectivos grupos. Apesar da necessidade de um treinamento para uso dos questionários, não foram identificadas dificuldades na aplicação com as outras presas. A participação de todas foi voluntária e a identificação nas fichas ficou a critério das presas.

A pesquisa foi autorizada pelos comitês de ética da PUC-Campinas ( $\mathrm{n}^{\circ}$ parecer: 2.209.813) e da Secretaria de Administração Penitenciária do estado de São Paulo (nº parecer: 2.438.660).

Para a avaliação dos dados, foi utilizado o método ANOVA para realizar a comparação de um domínio entre os níveis de uma variável, além da análise de Tukey, para a comparação par a par de todos os níveis da variável estudada.

\section{Resultados}

O local onde foi realizada a pesquisa é a única prisão que recebe mulheres na região de Campinas/SP, que é um dos maiores do interior do estado de São Paulo. A população carcerária total no início da pesquisa era de 440 presas (T0: $n=440$ ). Trata-se de um número que permaneceu variando durante todo o período da pesquisa, pois muitas presas estavam aguardando sentença, outras saíram em liberdade ou estavam apenas na 
unidade transitando entre estabelecimentos prisionais. Não foram consideradas presas que estavam em trânsito, somente a população fixa.

Apesar de toda a população fixa ser convidada a participar de forma voluntária, pois não havia critério de exclusão, o tamanho amostral final foi de $214(n=214)$, pois a maioria se recusou a participar $(51,3 \%)$. As justificativas foram a falta de interesse, receio de interferência no processo criminal, sentimento de falta de segurança sobre o sigilo ou ainda de não ter qualquer benefício decorrente da participação. A maioria nem apresentou justificativa da recusa.

A partir do questionário geral, foi possível traçar o perfil epidemiológico das detentas quanto à situação conjugal, etnia e escolaridade [Tabela 1].

Tabela 1 - Características sócio demográficas da população estudada ( $n=214$ )

\begin{tabular}{l|cc}
\hline Características & $\boldsymbol{n}$ & $\%$ \\
\hline Estado civil & 122 & $57.0 \%$ \\
Solteira & 77 & $35.9 \%$ \\
Casada & 8 & $3.73 \%$ \\
Separada & 7 & $3.27 \%$ \\
Viúva & $\mathbf{N}$ & $\mathbf{N}$ \\
Nível de escolaridade & 2 & $0.93 \%$ \\
Analfabeta & 104 & $48.60 \%$ \\
Ensino fundamental & 92 & $42.99 \%$ \\
Ensino médio & 11 & $5.14 \%$ \\
Faculdade (incompleto) & 3 & $1.40 \%$ \\
Faculdade (completo) & 2 & $0.93 \%$ \\
NA & &
\end{tabular}

NA: não se aplica 
A média de idade das presas foi de 32,4 anos (SD +/-10). A maioria respondeu ser solteira, de etnia branca seguida de parda e com baixa escolaridade (ensino fundamental incompleto). Esses achados são compatíveis com estudos prévios e indicativos de outros levantamentos realizados com a população de mulheres presas, que encontraram mais mulheres de baixa renda e não brancas $[\underline{9}, \underline{10}]$.

Segundo a escala WHOQOL-BREF [Tabela 2], nos quatro domínios estudados foram encontradas pontuação de nível "regular" (1 a 2,9) e "insatisfatória ou necessita melhorar" (3 a 3,9). O domínio referente ao meio ambiente recebeu a menor pontuação. Os domínios físico, psicológico e social classificaram-se na faixa "regular". Nenhum atingiu as

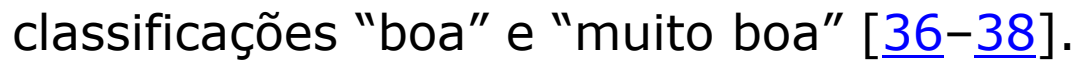

Tabela 2 - Pontuação para os domínios da escala WHOQOL-BREF

\begin{tabular}{c|ccc}
\hline Domínio & $\begin{array}{c}\text { Pontuação } \\
*\end{array}$ & Nível & Nível \\
\hline Físico & 3.04 & Regular & Regular \\
Psicológico & 3.34 & Regular & Regular \\
Social & 3.23 & Regular & Regular \\
Ambiental & 2.24 & Necessita melhorar & Necessita melhorar \\
\hline
\end{tabular}

*1 to $2.9=$ necessita melhorar; 3 to $3.9=$ regular; 4 to $4.9=$ bom; $5=$ muito bom

Essas pontuações indicam baixa qualidade de vida. Nível de pontuação semelhantes foram encontradas em populações acometidas por doenças crônicas e condições mórbidas ou incapacitantes [를 $\underline{33}]$. Apesar da baixa pontuação neste domínio, apenas $7,0 \%$ das presas recebiam tratamento psicológico e $10.7 \%$ recebiam tratamento psiquiátrico. Geralmente eram tratamentos pouco estruturados, com frequência irregular e com poucas opções de medicações psiquiátricas.

A pesquisa permitiu também avaliar a presença de pensamentos suicida e de tentativa de suicídio dentro da prisão, a qual mostrou níveis elevados.

Foram avaliadas dentro de cada domínio na escala de qualidade de vida, WHOQOL-BREF. No domínio 'psicológico" a pontuação foi baixa nesta 
escala para os dois quesitos, como seria esperado, além de apresentar diferença com valor estatístico significativo, conforme Tabela 3. As presas que já pensaram em suicídio têm uma pontuação média 1.66 menor no domínio 'psicológico" do que as que não pensaram. Faz parte da avaliação deste domínio itens como sentimentos positivos, pensar de forma positiva, conseguir aprender, capacidade de cognição, imagem corporal e aparência, entre outros.

Tabela 3 - Pensamento suicida, tentativa de suicídio e pontuação no WHOQOL-BREF

\begin{tabular}{l|ccc}
\hline & $N=214$ & $\begin{array}{c}\text { WHOQOL-brief } \\
\text { Domínio Psicológico } \\
\text { (media) }\end{array}$ & Value $p$ \\
\hline Pensamento suicida & $87(40.6 \%)$ & 3.47 & $* \mathbf{0 . 0 1 0 4}$ \\
Não & $84(39.2 \%)$ & 3.19 & \\
Sim & $43(20.1 \%)$ & 3.36 & \\
$* *$ NA & & & 0.1714 \\
Tentativa de & $95(44.4 \%)$ & 3.42 & \\
suicídio & $57(26.6 \%)$ & 3.25 & \\
Não & $62(28.9 \%)$ & 3.28 & \\
Sim & & & \\
**NA & & & \\
\hline
\end{tabular}

*Análise estatística da variável do domínio psicológico (Método ANOVA): $p<0,05$ **NA: Não se aplica

Foi também observado que "receber visitas" agiu como fator protetor para ideação suicida e tentativa de suicídio. Presas que recebiam visitas frequentes, além de apresentarem pontuação maior no nível domínio 'psicológico" da escala WHOQOL-BREF, tinham menos pensamentos suicidas ou tentativa de suicídio dentro da prisão. No grupo que não apresentou ideação suicida, $72,41 \%$ recebiam visitas regularmente, e no grupo que não tentou suicídio, $70,53 \%$ também recebiam visitas. A observação das visitas como fator de proteção chamou a atenção dos pesquisados, contudo, apesar da diferença perceptível, não houve significância estatística. 


\section{Discussão}

Trata-se de uma população em condição de vulnerabilidade e risco. Somase à condição de estar presa, a condição de ser do gênero feminino [이] . Os dados deste estudo reforçam a condição de vulnerabilidade. Tanto a baixa pontuação de Qualidade de Vida medida pela WHOQOL-BREF como a presença de pensamentos ou comportamento suicida.

Os dados relacionados ao suicídio contrastam bastante com os resultados de um estudo feito com a população geral do mesmo município, onde foi feito este estudo com a população prisional. Com um tamanho amostral de $538(n=538)$, ao longo da vida, $17,1 \%$ dos habitantes de 14 anos ou mais já "pensaram seriamente em pôr fim à vida", 4,8\% chegaram a planejar o ato, e $2,8 \%$ efetivamente tentaram o suicídio [40]. Ressaltam-se também estudos internacionais sobre prevalência de ideação suicida, que mostram valores de 2,1 a $18,5 \%$ de ideias suicidas ao longo da vida [41]. Comparativamente, nosso estudo encontrou valores muito acima dos dois estudos. A presença de pensamentos suicidas esteve presente em 39,2\% e a presença da tentativa de suicídio chegou a $26,6 \%$.

Sabe-se que história de tentativa de suicídio prévia e presença de transtornos mentais são os dois principais fatores de risco para o suicídio $[\underline{26}, \underline{42}]$, contudo, isolamento social, devido ao próprio confinamento e perda dos vínculos, estresse crônico, somados aos fatores anteriormente descritos, compõem um ambiente propenso ao adoecimento, mas passível de intervenções. Estudo comparando $o$ ato suicida entre homens e mulheres presas identificam maior vulnerabilidade das mulheres aos transtornos mentais e uso de drogas, além de serem mais vítimas de violência no ambiente prisional [43].

A significância desses dados chama a atenção para possíveis intervenções públicas, visto que o suicídio é uma morte passível de prevenção e com fatores de risco bastante elucidados [26, $\underline{40}$, 44]. Nesse sentido, intervenções específicas na área de saúde mental, como atendimento psiquiátrico e psicológico, podem reduzir o impacto desse padrão de comportamento neste ambiente, além de dar condições psíquicas para uma saída segura e favorável.

Em relação à pontuação de Qualidade de Vida medida pela WHOQOL-BREF, notadamente foi encontrado valores baixos em todos os domínios. Os resultados podem ser comparados com outros tipos de populações ou 
populações semelhantes com diferentes condições, como população de doentes crônicos ou em condições sociais de abandono [32-35].

Ter recebido visitas teve um impacto geral na saúde mental com pontuação média maior no domínio psicológico de Qualidade de Vida, além de menor frequência de pensamentos ou comportamento suicida entre as presas.

Na avaliação do domínio "psicológico" da escala WHOQOL-BREF, as presas que já pensaram em suicídio tinham uma pontuação média 1.66 menor no domínio 'psicológico" do que as que não pensaram. Considerando que este domínio avalia itens como sentimentos positivos, pensar de forma positiva, conseguir aprender, entre outros, este achado ilustra como o comportamento suicida não ocorre a partir de fatores isolados e sim dentro

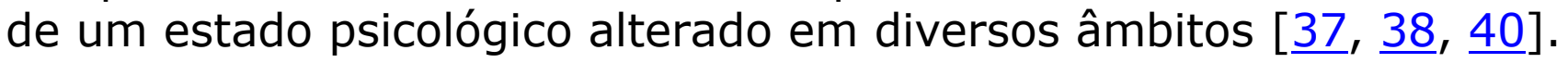

Apesar do que preconiza a regra 4 de Bangkok, discutida anteriormente, o que se observa no Brasil é uma concentração da população prisional feminina em poucas unidades, sendo comum que sejam localizadas longe de suas cidades de origem, onde residem seus familiares e amigos. A distância e as despesas com transporte agem como barreiras para as visitas [45]. O distanciamento da sociedade e de seus círculos afetivos cultiva um isolamento que pode ser preocupante $[\underline{46}, \underline{47}]$.

Outro ponto importante que limita as visitas são as questões de gênero. Enquanto as companheiras e esposas de homens presos continuam mantendo o vínculo, inclusive com visitas regulares, íntimas ou não, o contrário geralmente não acontece. As presas são rapidamente abandonadas por seus companheiros e maridos, seja pelo estigma da mulher infratora ou questões culturais, seja por iniciar novas relações afetivas [14].

Existem ainda outros entraves para a visita, que geralmente são resultantes de regras próprias de cada unidade prisional. Horários inviáveis de visita, em dias úteis, por exemplo, acabam por dificultar o acesso porque afetam dinâmicas de trabalho. Embora estejam previstas constitucionalmente, as visitas íntimas também ficam submetidas a regras locais, como, por exemplo, comprovação de união conjugal prévia ou casamento. Outro constrangimento é o fato de que parceiras do mesmo sexo biológico não têm permissão para realizar visita íntima [30]. 
Novamente a questão de gênero é muito proeminente: enquanto nos presídios masculinos as regras e condições para as visitas íntimas são bem menos rígidas e burocráticas, a desigualdade é justificada para elas pelos próprios funcionários e autoridades com a possibilidade de gravidez e supostas necessidades sexuais diferentes, como se mulheres tivesse uma percepção diferente de relações sexuais em comparação ao homem.

Nesse sentido, além da necessidade de atendimento de saúde mental já descrito acima, é fundamental criar ferramentas que facilitem as presas manterem seus vínculos externos, o apoio da família, de amigos e de outros relacionamentos. Todos têm papel significativo na Qualidade de Vida, como na redução do comportamento de suicídio [뇨, 49].

Apesar das limitações metodológicas, principalmente considerando que grande parte das presas não aceitaram participar da pesquisa, as quais poderiam ser até um grupo mais afetado negativamente por algum quadro depressivo ou serem mais suscetíveis a quadros suicidas, nossos dados apontam para uma necessidade de mudanças na forma de olhar o indivíduo preso do gênero feminino. Geralmente negligenciadas, pouco estudadas, além de abandonadas por famílias e parceiros.

\section{Conclusão}

O padrão de Qualidade de Vida encontrado na população estudada foi muito baixo, somente comparado a populações de doentes crônicos ou em situações sociais de abandono ou de risco. Foi encontrado também um padrão elevado de comportamento de risco para suicídio, muito acima do observado na população geral.

Receber visitas na prisão foi encontrado como um possível fator favorável para a saúde mental das presas, demonstrado pela pontuação média maior no domínio psicológico de Qualidade de Vida pela escala WHOQOL-BREF, além da diminuição da frequência de pensamento suicida ou de tentativas de suicídio.

Apesar de existirem regras que preconizem um modelo de assistência específico para a mulher encarcerada, a realidade prisional brasileira está longe de corresponder ao preconizado. Geralmente com condições que não facilitam o acesso a atendimento de saúde específico ao gênero, visitas de familiares, visitas íntimas e manutenção do vínculo familiar, fundamentais para a saúde mental e global da mulher. 
Sabendo da importância de fatores de risco para o suicídio, além da influência da saúde mental nas taxas de reincidência criminal, e considerando a relevância que as visitas apresentaram no presente estudo, tem-se um direcionamento para políticas públicas, seja para desburocratizar a visita às presas, seja para a criação de ferramentas que facilitem as visitas e reforcem seus vínculos familiares.

Por fim, esta população deverá receber abordagem ampla e específica para as suas características, tendo como finalidade, a reinserção social após a saída da prisão e diminuição da reincidência.

\section{Agradecimentos}

Os autores agradecem o Dr. Áureo Lopes, Procurador da República, e todos os funcionários da direção da Cadeia Feminina de Campinas/SP, em especial, Sra. Roseni Screpanti Braun e Sra. Sonia Aparecida, além de todas as detentas que aceitaram participar. 


\section{Referências}

1. Lopez-Rey M. The First U. N. Congress on the Prevention of Crime and the Treatment of Offenders. The Journal of Criminal Law, Criminology, and Police Science. 1957 Jan;47(5):526. https://doi.org/10.2307/1139020

2. Patterson RF. Our Part in the Evolution of Correctional Mental Health Care. The Journal of the American Academy of Psychiatry and the Law. 2018;46(2):7. https://doi.org/10.29158/JAAPL.003740-18

3. Dumont DM, Brockmann B, Dickman S, Alexander N, Rich JD. Public health and the epidemic of incarceration. Annu Rev Public Health. 2012 Apr;33:325-39. https://doi.org/10.1146/annurevpublhealth-031811-124614 PMid:22224880 - PMCid:PMC3329888

4. Lima LR, Oliveira AS. Minimum criminal law and the application of the Bangkok rules in the face of the incarceration of women. Revista Jurídica Direito, Sociedade e Justiça. [Internet]. 2018 Oct 19 [cited 2019 Feb 10];7(0). Available from:

http://periodicosonline.uems.br/index.php/RJDSJ/article/view/3096

5. Oliveira FS. Bangkok rules and female incarceration [Internet]. 2017 Apr [cited 2019 Feb 10]. Available from:

https://canalcienciascriminais.com.br/regras-de-bangkokencarceramento/

6. Brasil. Ministério da Justiça. Law 11.942/2009 [Internet]. Ministério da Justiça; 2009 [cited 2019 Feb 10]. Available from: http://www.planalto.gov.br/ccivil 03/ Ato20072010/2009/Lei/L11942.htm

7. Muniz CR, Bergo Leugi G, Alves AM. Women in the prison system: Why and how to understand their stories? RP3 - Revista de Pesquisa em Políticas Públicas [Internet]. 2018 Jan 24 [cited 2019 Feb $11] ; 11(2)$. Available from: http://periodicos.unb.br/index.php/rp3/article/view/26945 https://doi.org/10.18829/rp3.v11i2.26945 
8. Sidhu N, Candilis PJ. A Feminist Perspective for Forensic Practice. Journal of the American Academy of Psychiatry and the Law Online. 2018 Dec 1;46(4):438-46. https://doi.org/10.29158/JAAPL.003787$\underline{18}$

9. Moore LD, Elkavich A. Who's Using and Who's Doing Time: Incarceration, the War on Drugs, and Public Health. Am J Public Health. 2008 Sep;98(Suppl 1):S176-80. https://doi.org/10.2105/AJPH.98.Supplement 1.S176 PMid:18687610 - PMCid:PMC2518612

10. Walmsley R. World Prison Population List. 2010;15.

11. Rousseff D. Depen - Infopen Mulheres 2014. 2014 Jun p. 42.

12. Federal Government of Brazil. National Survey of Penitentiary Information 2014 - INFOPEN [Internet]. 2014 p. 42. Available from: http://www.justica.gov.br/news/estudo-traca-perfil-da-populacaopenitenciaria-feminina-no-brasil/relatorio-infopen-mulheres.pdf

13. Federal Government of Brazil. National Survey of Penitentiary Information 2018 - INFOPEN [Internet]. 2018 [cited 2019 Feb 12]. Available from:

http://depen.gov.br/DEPEN/depen/sisdepen/infopenmulheres/infopenmulheres arte 07-03-18.pdf/view

14. Soares Filho MM, Bueno PMMG. Demography, vulnerabilities and right to health of the Brazilian prison population. Ciênc saúde coletiva. 2016 Jul;21:1999-2010. https://doi.org/10.1590/141381232015217.24102015 - PMid:27383334

15. Bartlett $A$, Hollins $S$. Challenges and mental health needs of women in prison. Br J Psychiatry. 2018 Mar;212(3):134-6. https://doi.org/10.1192/bjp.2017.42 - PMid:29486822

16. Fazel S, Seewald K. Severe mental illness in 33,588 prisoners worldwide: systematic review and meta-regression analysis. $\mathrm{Br} \mathrm{J}$ Psychiatry. 2012 May;200(5):364-73.

https://doi.org/10.1192/bjp.bp.111.096370 - PMid:22550330 
17. Santos MV, Alves VH, Pereira AV, Rodrigues DP, Marchiori GRS, Guerra JVV. Mental health of incarcerated women in the State of Rio de Janeiro. Texto \& Contexto - Enfermagem [Internet]. 2017 [cited 2019 Feb 10];26(2). Available from:

http://www.scielo.br/scielo.php?script=sci arttext\&pid=S0104-

07072017000200314\&lng=en\&tIng =en

https://doi.org/10.1590/0104-07072017005980015

18. Audi CAF, Santiago SM, Andrade MGG, Francisco PMSB.

Common mental disorder among incarcerated women: a study on prevalence and associated factors. Ciência \& Saúde Coletiva. 2018 Nov;23(11):3587-96.

https://doi.org/10.1590/1413-812320182311.30372016 PMid:30427432

19. Shuford SH, Gjelsvik A, Clarke J, van den Berg JJ. Depression among Women Released from Prison or Jail in the United States. J Health Care Poor Underserved. 2018;29(3):914-29. https://doi.org/10.1353/hpu.2018.0068 - PMid:30122672

20. Opitz-Welke A, Bennefeldt-Kersten K, Konrad N, Welke J. Prison suicide in female detainees in Germany 2000-2013. Journal of Forensic and Legal Medicine. 2016 Nov 1;44:68-71. https://doi.org/10.1016/j.jflm.2016.08.016 - PMid:27636654

21. Opitz-Welke A, Bennefeld-Kersten K, Konrad N, Welke J. Prison suicides in Germany from 2000 to 2011. International Journal of Law and Psychiatry. 2013 Sep 1;36(5):386-9. https://doi.org/10.1016/j.ijlp.2013.06.018 - PMid:23850339

22. Federal Government of Brazil. National Survey of Penitentiary Information 2016 - INFOPEN [Internet]. 2016 [cited 2019 Feb 12]. Available from: http://depen.gov.br/DEPEN/noticias1 /noticias/infopen-levantamento-nacional-de-informacoespenitenciarias-2016

23. Moraes PAC, Dalgalarrondo P. Women imprisoned in São Paulo: mental health and religiosity. Jornal Brasileiro de Psiquiatria. 2006;55(1):50-6. https://doi.org/10.1590/S004720852006000100007 
24. WHO. WHO Suicide data [Internet]. 2016 [cited 2019 Jun 18]. Available from: https://www.who.int/healthtopics/suicide\#tab=tab 1

25. Rodrigues CD, Souza DS, Rodrigues HM, Konstantyner TCRO. Trends in suicide rates in Brazil from 1997 to 2015. Brazilian Journal of Psychiatry. 2019;0(0):1-9. https://doi.org/10.1590/1516-44462018-0230 - PMid:30785540 - PMCid:PMC6796812

26. Barbui $C$, Tansella M. Suicide prevention strategies and mental disorders. Epidemiology and Psychiatric Sciences. 2009 Sep;18(3):169-71. https://doi.org/10.1017/S1121189X00000415 PMid:20034191

27. Machado MFS, Leite CKS, Bando DH. Public Policies for Suicide Prevention in Brazil: a systematic review. 1. 2014 Dec 15;4(2):33456. https://doi.org/10.11606/issn.2237-1095.v4i2p334-356

28. Palmer EJ, Jinks M, Hatcher RM. Substance use, mental health, and relationships: A comparison of male and female offenders serving community sentences. International Journal of Law and Psychiatry. 2010 Mar 1;33(2):89-93.

https://doi.org/10.1016/j.ijlp.2009.12.007 - PMid:20036007

29. Sered SS, Norton-Hawk M. Women on the Institutional Circuit: A 9-Year Qualitative Study. J Correct Health Care. 2019 Jan 7;1078345818819811. https://doi.org/10.1177/1078345818819811 PMid:30616433

30. Oliveira M, Santos AF. Gender inequality in the prison system: considerations about barriers to visits and intimate visits to incarcerated women. Caderno Espaço Feminino [Internet]. 2012 Dec 18 [cited 2019 Feb 10];25(1). Available from:

http://www.seer.ufu.br/index.php/neguem/article/view/15095

31. WHO Division of Mental Health and Prevention of Substance Abuse. WHOQOL: measuring quality of life. 1997 [cited 2019 Jul 2]; Available from: https://apps.who.int/iris/handle/10665/63482 
32. Ginieri-Coccossis M, Triantafillou E, Papanikolaou N, Baker R, Antoniou C, Skevington SM, Christodoulou GN. Quality of life and depression in chronic sexually transmitted infections in UK and Greece: The use of WHOQOL-HIV/STI BREF. Psychiatriki. 2018 Sep;29(3):209-19.

https://doi.org/10.22365/jpsych.2018.293.209 - PMid:30605425

\section{Jesus NM, Souza GF, Mendes-Rodrigues C, Almeida Neto OP,} Rodrigues DDM, Cunha CM. Quality of life of individuals with chronic kidney disease on dialysis. Brazilian Journal of Nephrology [Internet]. 2019 [cited 2019 Jun 3];(ahead). Available from: http://www.scielo.br/scielo.php?script=sci abstract\&pid=S010128002019005004104\&lng=en\&nrm=iso\&tIng=en https://doi.org/10.1590/2175-8239-jbn-2018-0152 PMid:30720851 - PMCid:PMC6788844

34. Redko C, Rogers N, Bule L, Siad H, Choh A. Development and validation of the Somali WHOQOL-BREF among refugees living in the USA. Qual Life Res. 2015 Jun;24(6):1503-13. https://doi.org/10.1007/s11136-014-0877-3 - PMid:25429823

35. Alves JC, Bassitt DP. Quality of life and functional capacity of elderly women with knee osteoarthritis. Einstein (São Paulo). 2013 Jun;11(2):209-15. https://doi.org/10.1590/S1679-

45082013000200013 - PMid:23843063 - PMCid:PMC4872896

36. Bonomi AE, Patrick DL, Bushnell DM, Martin M. Validation of the United States' version of the World Health Organization Quality of Life (WHOQOL) instrument. J Clin Epidemiol. 2000 Jan;53(1):1-12. https://doi.org/10.1016/S0895-4356(99)00123-7

37. Fleck MP, Louzada S, Xavier M, Chachamovich E, Vieira G, Santos L, Pinzon V. Application of the Portuguese version of the abbreviated instrument of quality life WHOQOL-BREF. Revista de Saúde Pública. 2000 Apr;34(2):178-83. https://doi.org/10.1590/S0034-89102000000200012 PMid: 10881154

38. Fleck MP. The World Health Organization quality of life assessment tool (WHOQOL-100): characteristics and perspectives. 
Ciência \& Saúde Coletiva. 2000;5(1):33-8.

https://doi.org/10.1590/S1413-81232000000100004

39. Santos BRM, Rezende VA. Sistema carcerário feminino: uma análise das políticas públicas de segurança com base em um estudo local. Cad EBAPEBR. 2020 Oct 16;18:583-94.

https://doi.org/10.1590/1679-395120190034

40. Botega NJ, Marín-León L, Oliveira HB, Barros MBA, Silva VF, Dalgalarrondo $P$. Prevalence of suicidal ideation, suicide plans, and attempted suicide: a population-based survey in Campinas, São Paulo State, Brazil. Cadernos de Saúde Pública. 2009 Dec;25(12):2632-8. https://doi.org/10.1590/S0102311X2009001200010 - PMid:20191154

41. Suominen K, Isometsä E, Suokas J, Haukka J, Achte K, Lönnqvist J. Completed suicide after a suicide attempt: a 37-year follow-up study. Am J Psychiatry. 2004 Mar;161(3):562-3. https://doi.org/10.1176/appi.ajp.161.3.562 - PMid:14992984

42. Botega NJ, Werlang BG, Cais CF da S, Macedo MMK. Prevention of suicidal behavior. Psico. 2006;37(3):5. https://psycnet.apa.org/record/2007-11290-001

43. Mennicke A, Daniels K, Rizo CF. Suicide Completion Among Incarcerated Women. J Correct Health Care. 2021;14-22. https://doi.org/10.1089/jchc.18.12.0070 - PMid:34232762

44. Andersen UA, Andersen M, Rosholm JU, Gram LF. Contacts to the health care system prior to suicide: a comprehensive analysis using registers for general and psychiatric hospital admissions, contacts to general practitioners and practising specialists and drug prescriptions. Acta Psychiatr Scand. 2000 Aug;102(2):126-34. https://doi.org/10.1034/j.1600-0447.2000.102002126.x PMid: 10937785

45. Wolff N, Shi J. Childhood and Adult Trauma Experiences of Incarcerated Persons and Their Relationship to Adult Behavioral Health Problems and Treatment. International Journal of Environmental Research and Public Health. 2012 May;9(5):1908-26. 
https://doi.org/10.3390/ijerph9051908 - PMid:22754481 PMCid:PMC3386595

46. Bonnie E, Carlson, N. Inmates and their Families: Conjugal Visits, Family Contact, and Family Functioning. [Internet]. 1997 [cited 2019 Feb 10]. Available from:

https://journals.sagepub.com/doi/10.1177/0093854891018003005

47. Hensley C, Koscheski M, Tewksbury R. Does Participation in Conjugal Visitations Reduce Prison Violence in Mississippi? An Exploratory Study. Criminal Justice Review. 2002 May 1;27(1):5265. https://doi.org/10.1177/073401680202700104

48. Hegerl U. Prevention of suicidal behavior. Dialogues Clin Neurosci. 2016 Jun;18(2):183-90.

https://doi.org/10.31887/DCNS.2016.18.2/uhegerl PMid:27489458 - PMCid:PMC4969705

49. Marzano L, Hawton K, Rivlin A, Smith EN, Piper M, Fazel S. Prevention of Suicidal Behavior in Prisons. Crisis. 2016;37(5):32334. https://doi.org/10.1027/0227-5910/a000394 - PMid:27278569 PMCid:PMC5120691 
Eduardo Henrique Teixeira

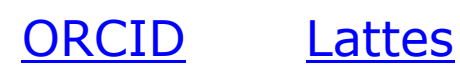

Rogério Gomes da Silva Júnior

$\underline{\text { ORCID Lattes }}$

Giuliana Perrotte

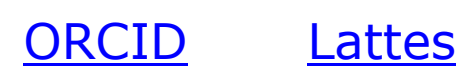

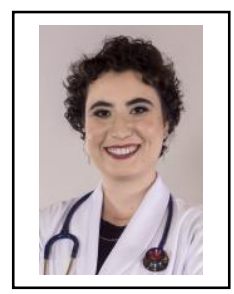

Maria Lígia de Carvalho Solssia

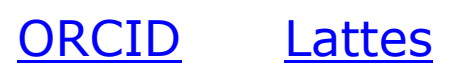

